\title{
Cáncer de mama
}

\author{
María Patricia Moll Agudelo ; Francisco Eugenio Gómez Botero*; Isabel Cristina Ruiz Buitrago**; \\ Gildardo Gallego Noreña***; Jaime Uribe Duque****
}

\section{RESUMEN}

INTRODUCCION: El cáncer de mama compite por el primer puesto con el cáncer de cérvix. Entidad frecuente en nuestro medio, lo cual está lejos de las políticas del Ministerio de Salud.

OBJETIVO: Determinar incidencia del cáncer de mama en el departamento de Antioquia en el período primero de enero de 1986 y el 31 de diciembre de 1991.

MATERIAL Y METODOS: Estudio retrospectivo longitudinal, se recopilan datos, previo formulario a través de informes de Patología con confirmación histológica de cáncer de mama de laboratorios públicos y privados.

RESULTADOS: Total de casos recopilados 2.915. La tasa de incidencia para cinco años fue 195, 61 casos por 100.000 mujeres, ascenso creciente a medida que aumenta la edad.

Indisciplina médica y pésimo diligenciamiento de la solicitud de estudio de patología.

CONCLUSIONES: Primera investigación de este tipo en Colombia. Se recomienda crear comités de patología mamaria en hospitales, crear red de información a nivel regional y central, fomentar el autoexamen mamario y sugerir mamografía para grupos de riesgo.

PALABRAS CLAVES: Incidencia, cáncer de mama.

\section{SUMMARY}

INTRODUCTION: The breast cancer compete by first place with cervix cancer: the statal politics are out of this problem.

OBJETIVE: Determine breast cancer incidence in Antioquia departament from 1 - I- 1986 to 31 - XII - 1991.

MATERIAL AND METHODS: Restrospective longitudinal study, recopilation of information by formularies previous throught private and publics pathologic registers with histologic confirmation breast cancer.

RESULTS: All cases was 2.915, incidence tase per five years was 191.61 cases per 100.000 women. Fifty six per cent are from metro politan area. 534,23 cases per 100.000 women was the higher incidence in decade sixth year old; its found increase as age is elevated. $93 \%$ was ductal infiltrative breast cancer.

Default diligence of solicitude of pathological studies. Lack of discipline of phicicians.

Its recommended create committee of breast pathologie in hospitals and create information red to regional and central level, beside teaching women examen her breasts. Prescribe mamografie to risk group.

KEY WORDS: Incidence, breast cancer.

\section{Introducción}

El cáncer de mama es una entidad que compite con el cáncer de cuello uterino por ocupar el primer lugar en Colombia como causa de morbimortalidad en la mujer. Se

* Residentes Tercer año de Obstetricia y Ginecología.

** Médica Epidemióloga. Servicio Seccional de Salud de Antioquia.

*** Médico Ginecólogo Oncólogo. Profesor Titular Universidad de Antioquia.

****Médico Ginecólogo Oncólogo. Profesor Titular Universidad de Antioquia. Centro para Estudios de la Salud CES. descubre cada vez más en mujeres jóvenes, las cuales están en plena actividad productiva, causa la muerte en muchas de ellas, dejando hijos menores y familias enteras en un verdadero desequilibrio social.

Esta patología es una prioridad nacional, está lejos de las políticas de salud. No existen campañas de prevención, promoción, diagnóstico, tratamiento y seguimiento adecuado. Basado en estos conceptos podemos plantear algunos interrogantes que nos sirvan de reflexión y motivar la investigación: 
1. ¿Cuál es el comportamiento real del carcinoma de mama en el país?

2. ¿Existen programas de prevención y diagnóstico pre$\mathrm{coz}$ ?

3. ¿El tratamiento es eficaz?

4. ¿Cuál es la actitud de los Servicios Seccionales de Salud y el Ministerio?

5. Existe soporte estatal para financiar el tratamiento. Muchos de estos interrogantes seguirán sin resolverse.

La responsabilidad que se tiene como profesionales del área de la salud directamente vinculados a la atención de este grupo de pacientes, hace que exista una motivación para conocer en nuestro medio la realidad del comportamiento de estas enfermedades y de vincular a las diferentes entidades con servicio de patología y a los patólogos particulares para hacer un frente común que logre establecer una red de diagnóstico y tratamiento para cáncer de mama.

Se desea investigar sobre la frecuencia real del cáncer de mama por las siguientes razones:

1. Existe en el país y en el departamento una carencia de información que permita identificar con claridad la frecuencia real del cáncer de mama.

2. No existe un sistema de vigilancia epidemiológica en cáncer que permita una aproximación al conocimiento de los factores de riesgo, frecuencia, mortalidad, precocidad en el diagnóstico etc.

3. El carcinoma de mama dada su gravedad por ser una enfermedad sistémica, su diagnóstico es generalmente tardío por lo que se hace necesario conocer su incidencia y montar un sistema de vigilancia epidemiológica que garantice un diagnóstico precoz y una red de atención que funcione adecuadamente.

4. Se pretende con esta investigación tener un punto de partida en el conocimiento de esta patología para proponer un sistema de vigilancia epidemiológica en cáncer, tomando como fuente de información esencial los resultados de patología institucionales y particulares y estructurar una red de diagnóstico y tratamiento que garantice la calidad de la atención a este grupo poblacional.

5. Es prioridad para el Servicio Seccional de Salud de Antioquia esta línea de investigación.

\section{Objetivo general}

Determinar la incidencia de cáncer de mama en el Departamento de Antioquia en el período comprendido entre el 1o. de enero de 1986 y el 31 de diciembre de 1991.

Como objetivos específicos: identificar casos de carcinoma in situ e invasor, grupos de edad, procedencia, tipos histológicos más frecuentes e identificarlocalización topográfica del cáncer de mama.

\section{Material y métodos}

Se realizó un estudio descriptivo, retrospectivo y longitudinal con la participación de los patólogos, ginecólogos y oncólogos del departamento en el período del 1o. de enero de 1986 y el 31 de diciembre de 1991.

La información se recopiló del reporte anatomopatológico de las biopsias tomadas y el espécimen quirúrgico de mama, leídas por cada uno de los patólogos particulares y/ o vinculados a las instituciones del Departamento de Antioquia.

\section{Análisis de resultados}

El total de casos reportados en el Departamento de Antioquia entre los años 1986 y 1991 fueron 2.915; con una tasa de prevalencia para los cinco años de 195.61 casos por 100.000 mujeres, el $56 \%$ (1.628) procedían del Area Metropolitana (cuadro 1).

\section{Cuadro 1 \\ CANCER DE MAMA. DEPARTAMENTO DE ANTIOQUIA 1986-1991}

Distribución según procedencia

\begin{tabular}{|lrr|}
\hline $\begin{array}{l}\text { Ca de mama } \\
\text { Procedencia }\end{array}$ & Número & \% \\
\hline Area Metropolitana & 1628 & 56 \\
Urabá & 17 & 0.6 \\
Occidente & 14 & 0.4 \\
Suroeste & 56 & 1.9 \\
Bajo Cauca & 18 & 0.6 \\
Meseta & 28 & 0.9 \\
Nordeste & 21 & 0.7 \\
Magdalena Medio & 8 & 0.3 \\
Oriente & 63 & 2.4 \\
Sin datos & 1.029 & 36.0 \\
Total & 2.888 & $100 \%$ \\
\hline
\end{tabular}

Fuente: Archivos de la investigación.

$$
\text { Cuadro } 2
$$

CANCER DE MAMA. DEPARTAMENTO DE ANTIOQUIA 1986-1991

Tasa específica por edad

\begin{tabular}{|lcr|}
\hline Edad & Número & Tasa \\
\hline $15-19$ & 4 & 1.69 \\
$20-29$ & 84 & 18.29 \\
$30-39$ & 325 & 105.72 \\
$40-49$ & 616 & 332.54 \\
$50-59$ & 601 & 454.37 \\
60 y más & 730 & 534.23 \\
Sin dato & 555 & -195.61 \\
Total & 2.915 & 195.6 \\
\hline
\end{tabular}

Fuente: Registros de la investigación.

Cuadro 3

CANCER DE MAMA. DEPARTAMENTO DE ANTIOQUIA 1986-1991

Distribución por histología y grado de invasión

\begin{tabular}{|lr|rr|rr|}
\hline Invasión & \multicolumn{2}{c|}{ In situ } & \multicolumn{3}{c|}{ Infiltrante } \\
\hline Histología & No. & $\%$ & No. & $\%$ & Total \\
\hline Ca ductal & 195 & 6.4 & 2706 & 88.3 & 2901 \\
Ca lobular & 43 & 1.4 & 88 & 2.9 & 131 \\
Sarcoma & 0 & 0 & 30 & 1.0 & 30 \\
Total & 238 & 7.8 & 2824 & 92.2 & 3062 \\
\hline
\end{tabular}

Fuente: Registros de la investigación. 
En la distribución según edad se encontró una tasa de prevalencia mayor en el grupo etáreo de 60 o más años con 534.23 casos por 100.000 mujeres (730); observándose en general un ascenso en las tasas de incidencia a medida que aumenta la edad. El 19,3\% (555) de los casos del estudio carecían del dato de la edad en los registros de la solicitud de biopsias (cuadro 2, gráfica 1).

\section{Gráfico 1}

CANCER DE MAMA. INCIDENCIA SEGUN EDAD

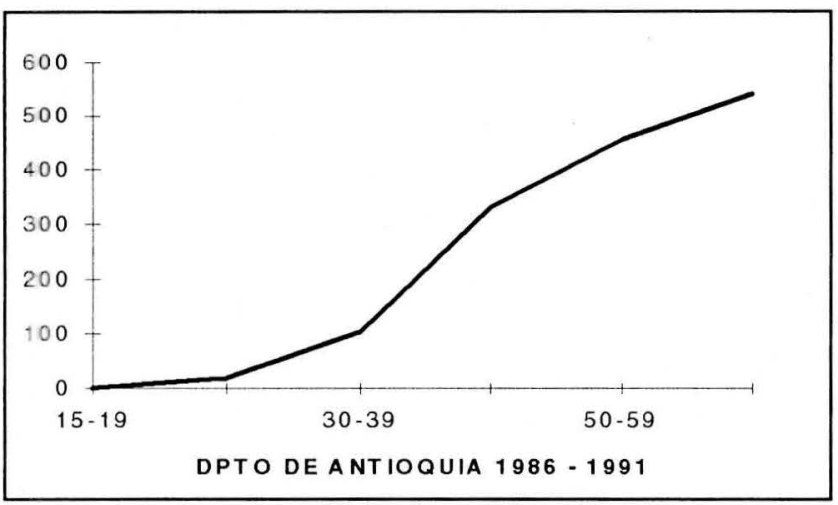

En cuanto al tipo histológico: entre 3.062 especímenes reportados el 92,9\% (2.824) fueron del tipo infiltrante, presentándose con mayor frecuencia el cáncer ductal con el 88,3\% (2.706); tan sólo el 7,8\% (238) de los carcinomas de mama reportados fueron in situ (cuadro 3 ).

La prevalencia observada por año desde 1986 a 1991 del cáncer de mama, tuvo una variación por año de 25.5 a 35.57 casos $/ 100.000$ mujeres, siendo el año de 1989 el que representó mayor prevalencia (cuadro 4, gráfica 2).

Cuadro 4

\section{CANCER DE MAMA. DEPARTAMENTO DE ANTIOQUIA 1986-1991}

Tasa de incidencia por año

\begin{tabular}{|lcc|}
\hline Ca de mama & No. & $\begin{array}{c}\text { Tasa } \\
\mathbf{X} \mathbf{1 0 0 . 0 0 0} \\
\text { Mujeres }\end{array}$ \\
\hline Año & & 25.50 \\
1986 & 399 & 29.95 \\
1987 & 447 & 35.03 \\
1988 & 488 & 35.57 \\
1989 & 504 & 34.60 \\
1990 & 525 & 35.46 \\
1991 & 552 & \\
\hline
\end{tabular}

Fuente: Registros de la investigación.

La distribución según la localización del cáncer de mama fue: lado derecho $940(32,2 \%)$ y el izquierdo 987 $(33,8 \%)$, llama la atención que casi igual número de casos $980(32,2 \%)$ no tienen consignado la localización del cáncer en los registros de solicitud de biopsia (cuadro 5). En los cuadrantes superiores externos se encontraron 509 casos $(16 \%)$ el mayor número, seguido del cuadrante superior interno con 186 casos $(5,8 \%)$.
Gráfico 2

CANCER DE MAMA. TASA DE INCIDENCIA POR AÑO

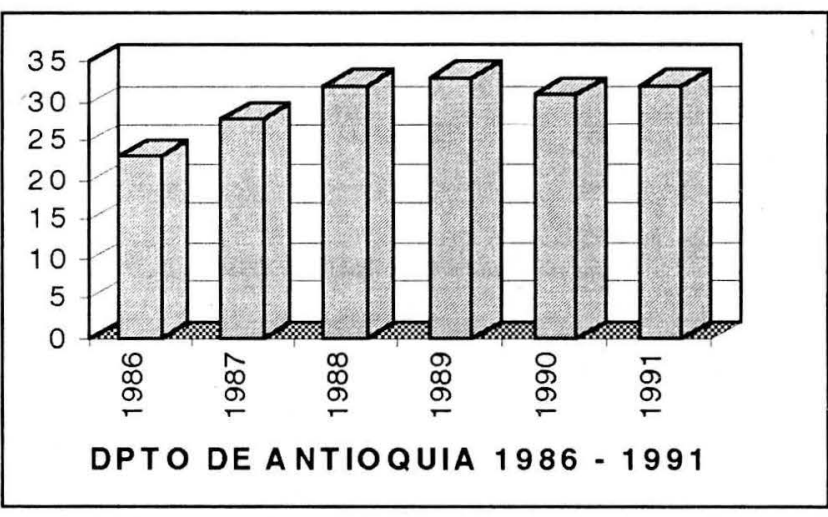

\section{Cuadro 5 \\ CANCER DE MAMA. DEPARTAMENTO DE ANTIOQUIA 1986-1991}

Distribución según localización

\begin{tabular}{|lrr|}
\hline $\begin{array}{l}\text { Ca de mama } \\
\text { localización }\end{array}$ & No. & \% \\
\hline Mama derecha & 940 & 32.2 \\
Mama izquierda & 987 & 33.8 \\
Bilateral & 24 & 0.7 \\
Sin dato & 980 & 33.3 \\
Total & 2931 & 100.0 \\
\hline
\end{tabular}

Fuente: Registros de la investigación.

Es importante anotar que el 1,3\% (42) casos tenían localizado el tumor en toda la mama, además hay un gran número de casos 62,1\% (1980) sin registro de localización del tumor en el informe de patología (cuadro 6).

Del total de casos de carcinoma de mama 2.915, cinco se presentaron en hombres con una tasa de incidencia de 0,24 por 100.000 hombres para el período, todos ellos del tipo histológico ductal infiltrante y con una distribución por edades de $80 \%$ en mayores de 40 años (cuadro 7).

\section{Discusión}

El cáncer de mama es en la actualidad el tumor maligno más frecuente entre las mujeres blancas de los países industrializados. La Sociedad Americana de Cáncer publicó en 1980 datos que demuestran que el cáncer de mama es la causa más frecuente de muerte en mujeres de 39 a 44 años de edad (13).

En nuestro país este cáncer compite con el de cuello uterino por el primer lugar. No existen en Colombia estadísticas bien establecidas frente a la prevalencia e incidencia del cáncer de mama, pero si existen estudios que mencionan aspectos diagnósticos importantes como la punción con aguja fina $(5,22,25)$.

Del total de encuestas tabuladas se encontraron 2.915 pacientes con ca de mama en el departamento de Antioquia en el período comprendido entre 1986 y 1991, lo que daría 


\section{Cuadro 6 \\ CANCER DE MAMA. DEPARTAMENTO DE ANTIOQUIA 1986-1991}

Distribución según cuadrante

\begin{tabular}{|lrr|}
\hline \begin{tabular}{lrr|} 
Ca de mama \\
Cuadrantes
\end{tabular} & No. & $\%$ \\
\hline Supero externo & 509 & 16.0 \\
Supero interno & 186 & 5.8 \\
Infero externo & 151 & 4.7 \\
Infero interno & 66 & 2.0 \\
Supero ext. e int. & 45 & 1.4 \\
Supero int. e infero int. & 9 & 0.3 \\
Supero ext. y supero int. & 51 & 1.6 \\
Infero ext. e infero int. & 21 & 0.7 \\
Retroareolar & 80 & 2.5 \\
Toda la mama & 42 & 1.3 \\
Multicéntrico & 37 & 1.2 \\
Sin datos & 1980 & 62.1 \\
Total & 3177 & 99.5 \\
\hline
\end{tabular}

Fuente: Registros de la investigación.

Cuadro 7

CARCINOMA DE MAMA EN HOMBRES. DEPARTAMENTO DE ANTIOQUIA 1986-1991

Distribución según edad

\begin{tabular}{|ccc|}
\hline Edad & No. & $\%$ \\
\hline$<45$ & 1 & 20 \\
$>46$ & 4 & 80 \\
Total & 5 & 100 \\
\hline
\end{tabular}

Fuente: Registros de la investigación.

una incidencia acumulada de 195.61 casos por 100.000 mujeres (cuadro 2).

El cáncer de mama es una enfermedad predominante del mundo occidental donde se ha demostrado que es 1,5 veces mayor que en los países orientales, tal como se aprecia en las estadísticas de la Organización Mundial de la Salud que desde 1945 publica las estadísticas de 45 países $(3,12,17)$.

La incidencia del cáncer de mama presenta un lento aumento en los Estados Unidos y se estima que en 1991 se diagnosticó dicho tumor en 175.000 mujeres (9). Para 1992 fueron diagnosticadas 180.000 y se estimo que 46.000 de estas murieron de la enfermedad (27). En Europa se encuentran incidencias de: Suiza 70,6, Suecia 52,4 y en Canadá de 80 casos x 100.000 mujeres $(12,14)$.

En países subdesarrollados como Polonia y Nigeria se informa una incidencia de $15.3 \times 100.000$ mujeres, esto posiblemente se deba a una información en salud fragmentada e incompleta o por dificultades en la atención en salud propia de estos países. En Colombia se piensa que la situación es similar. En esta investigación una de las primeras que muestra el comportamiento del cáncer de mama en el departamento de Antioquia. Hasta el momento la información en Colombia sobre carcinoma de mamaha sido la presentada por el Instituto Nacional de Cancerología con sede en Santa Fe de Bogotá que dispone de una información parcial del país. Esta investigación es un aporte actualizado del comportamiento de dicho cáncer en el departamento.

El Area Metropolitana en Antioquia demostró una incidencia para el período mayor con respecto a otras regiones, con 1.628 casos (56\%); siguiéndole en frecuencia el oriente con 63 casos $(2,4 \%)$ y suroeste con 56 casos $(1,9 \%)$. Esta gran diferencia podría tener explicación si se considera que el Area Metropolitana es un sitio de referencia de las demás regiones, donde se ubican el mayor número de especialistas y existe mayor facilidad para el diagnóstico y el tratamiento.

Clásicamente se ha descrito una curva bimodal en el comportamiento del ca de mama referente a la edad, con la aparición de un pico precoz premenopáusico influido por estrógenos ováricos y otro tardío por acción de estrógenos suprarrenales y de sustitución (12). Cuando se compara la edad de aparición del cáncer de mama en los diferentes países se observa que en aquellos que tienen una alta incidencia (como en los Estados Unidos, Canadá, Dinamarca y Reino Unido) se observa un aumento de frecuencia después de la menopausia pero menos notorio en las mujeres jóvenes. Mientras que en países con baja incidencia (Grecia, Japón y Polonia) ésta disminuye después de la menopausia (20). En Antioquia se encontró una tasa de incidencia máxima en el grupo etáreo de 60 y más años de 534.23 casos por 100.00 mujeres. Se observo un ascenso constante en la tasa por edades hasta los 60 años; no se pudo precisar el descenso postmenopáusico ya que no se disponen de datos poblacionales entre los otros grupos de edad 71-80, 81 y más (cuadro 2). En países del Caribe de habla inglesa el cáncer de útero y mama tienen proporción igual de defunción en el grupo etáreo de 35 a 64 años (21).

De los 3.062 casos de ca de mama el tipo histológico más frecuente fue el ductal infiltrante con 2.702 casos que corresponde al $88,3 \%$. Esto se acerca a lo demostrado en otros estudios como el de Fisher, B $(20,28)$ quien encuentra este tipo histológico en el $50 \%$ de todos los cáncer de mama y el estudio de Baker en 1982 que informa de 75 al $80 \%(8,15)$.

La variedad de carcinoma lobulillar infiltrante le sigue en frecuencia con reportes sólo entre el 5 y $15 \%$ de los carcinomas de mama según la serie y con frecuencia son multicéntricos (15-28). En esta investigación se reportaron 88 casos $(2,9 \%)$ de lobulillar infiltrante; siendo más frecuente el ductal in situ con 195 casos $(6,4 \%)$ que en la literatura representa el 3-5\%de todos los cáncer de mama (cuadro 3).

El tamaño del tumor, nódulos axilares metastásicos, la presencia de receptores hormonales, edad, localización topográfica y otros se consideran factores pronósti$\cos (16)$. Se ha demostrado por ejemplo que el ca ductal infiltrante tiene un pobre pronóstico y que comúnmente es metastásico a los ganglios axilares (9-15) los tipos puros mucinosos, tubular o papilar sin metástasis a la axila confieren un pronóstico relativamente favorable, así como el cáncer medular (10-23). Actualmente está en discusión si el tratamiento varia con el tipo histológico. 
La incidencia del cáncer de mama por año mostró una variación importante con una tasa de 25,5 a 35,45 casos por 100.000 mujeres, se estabilizó en los tres últimos años (cuadro 4). Se esperaría una incidencia en aumento anual, si las coberturas de salud en el diagnóstico y búsqueda activa en el Departamento de Antioquia fueran mejorando año a año. Actualmente no existen campañas o estrategias para tal fin. Por el contrario la estabilidad de la incidencia en los últimos años en esta investigación, hace pensar en la pobre búsqueda del carcinoma de mama por el personal de salud, ausencia de una red de diagnóstico y de información que garantice una detección precoz del carcinoma de mama.

Se ha demostrado que con programas de selección de mujeres alto riesgo se hace cuatro veces más diagnóstico que en los programas con pacientes no seleccionados (14). Estos programas podrían incluir la autoexploración, la exploración clínica periódica por el especialista y la mamografía con la cual se puede detectar dos años antes de ser palpable (2). El plan de seguridad sanitario de Nueva York con sus estudios indican que estos tres métodos contribuyen a reducir la mortalidad del cáncer de mama (3).

El Breast Cáncer Detection Demostration Project (BCDDP) demostró que $91 \%$ de los cánceres se detectaron por mamografía; otros grandes estudios han demostrado una sensibilidad del 86 al 95\% (1). La mamografía permite detectar tumores más tempranos y con menos metástasis. Así el 97\% de los cánceres mínimos se detectan por este método y sólo $33 \%$ se identifican en la exploración física segủn estudios de Cooper (4).

La creencia de que otros métodos podrían ser adecuados es un error, la BCDDP demostró que la tomografía tiene una sensibilidad de sólo $42 \%$, la transiluminación de $30 \%$; la tomografía computarizada, la resonancia magnética y el ultrasonido no pueden localizar de forma confiable las microcalcificaciones del cáncer mamario (2-4). Se ha encontradopredominio del cáncer, por la mama izquierda sin que exista una explicación a este hallazgo, en la investigación no se encontró diferencia siendo $32,2 \%$ en mama derecha y $33,8 \%$ en la mama izquierda (cuadro 5).

Las pacientes tratadas de cáncer de mama tienen un riesgo entre tres y seis veces mayor de desarrollar un cáncer en la otra mama (8). En este estudio sólo se conoce que 24 casos $(0,7 \%)$ presentaron bilateralidad. Se desconoce de que tipo histológico (cuadro 5).

Se ha reportado en la literatura que el carcinoma de mama se localiza con mayor frecuencia en el cuadrante superior externo en un $45 \%$ de los casos, seguido de la zona subareolar $20 \%$, cuadrante superior externo $15 \%$ y ambos cuadrantes inferiores con un $10 \%$ cada uno (3). Este estudio demostró un $16 \%$ de los casos localizados en el cuadrante superior externo como el principal sitio de ubicación del cáncer de mama, seguido del cuadrante superior e interno con un 5,8\% (cuadro 6).

Se encontró un importante porcentaje de casos 1.980 $(62,1 \%)$ sin datos en los registros de solicitud de biopsia que da la impresión de que la localización topográfica es de poca importancia para el clínico y que aporta poco al patólogo lo cual no es cierto, porque el carcinoma de mama ubicado en los cuadrantes internos y subareolar tienen un pronóstico más pobre pues hacen con más frecuencia diseminación a ganglios mamarios internos dificultando así su abordaje quirúrgico. Los tumores de la mama situados en los cuadrantes inferiores derechos tienen peor pronóstico por el peligro de diseminación al hígado, los de mejor pronóstico son los situados en los cuadrantes externos (13).

De los 2.915 pacientes con cáncer de mama encontrados en esta investigación, cinco fueron hombres que corresponde a una tasa de incidencia para el período de 0,24 casos por 100.000 hombres: lo que coincide con los estudios norteamericanos que informan que el carcinoma de mama en hombres constituye sólo el $1 \%$ o menos de la frecuencia del cáncer.

El carcinoma de mama es sólo el 1,5\% de todos los tumores malignos en hombre. Aquí se observaron cuatro casos $(80 \%)$ en mayores de 40 años y sólo 1 caso $(20 \%)$ menor de 40 años, (cuadro 7) lo que concuerda con lo publicado (24).

La edad promedio de detección del carcinoma de mama en hombres es de 60 años, 6 a 11 años más tarde que en la mujer. Sólo $1 \%$ de cáncer de mama en hombres ocurre antes de los 30 años y $6 \%$ antes de los 40 años (7). La curva bimodal de distribución de edad para el carcinoma de mamaen la mujer no se ha visto en el hombre. Hay una leve e inexplicable predilección por la mama izquierda en el hombre similar que en las mujeres; cuatro de los cinco casos reportados en hombres se localizaron en la mama izquierda.

Los tipos histológicos encontrados en hombres son semejantes al de la mujer excepto el carcinoma lobular in situ que sólo se ha reportado escasamente (6). Los cinco casos reportados en este estudio fueron del tipo histológico ductal infiltrante que es el más frecuente, ya que incluye del 50 y $80 \%$ de todos los cáncer de mama según la serie, como se mencionó antes.

\section{Conclusiones}

1. Se observo a través de la investigación el pésimo diligenciamiento de los datos de solicitud de estudio histopatológico, lo cual dificulta el seguimiento y tratamiento de dichas pacientes y distorsiona el análisis de la información.

2. La incidencia acumulada del cáncer de mama en Antioquia entre los años de 1986 y 1991 fue de 195,61 casos por 100.000 mujeres.

3. Se observo un ascenso constante en la incidencia del cáncer de mama por grupos etáreos, siendo la edad de mayor incidencia el de 60 y más años, con una tasa de 534,23 casos por 100.000 mujeres.

4. El carcinoma ductal infiltrante fue el tipo histológico más frecuente entre los carcinomas de mama con un $88,3 \%$, hallazgo que concuerda con la literatura mundial.

5. Se encontró una notoria diferencia en la distribución de cáncer de mama según la procedencia con $56 \%$ de los casos encontrados en el área metropolitana y de 2,4 a $0,3 \%$ en otras regiones; atribuido posiblemente al desarrollo de la infraestructura para el diagnóstico y tratamiento.

6. La incidencia del cáncer de mama por año presentó un aumento en los tres primeros años, de 25,5 a 35,03 
casos por 100.000 mujeres y estabilizándose en los últimos tres.

7. En el $62 \%$ de los casos la localización del cáncer de mama no fue especificada en los registros de patología. De los registrados, el cuadrante supero-externo fue más frecuente $(16 \%)$.

8. La incidencia de cáncer de mama en hombres fue de 0,24 casos por 100.000 hombres, el $80 \%$ de los casos en mayores de 40 años.

\section{Recomendaciones}

1. Crear una estructura ágil para el diagnóstico, remisión oportuna, tratamiento y seguimiento de las pacientes, mejorando la dotación de los centros regionales en cuanto a personal capacitado y equipos.

2. Elaborar un registro único tanto institucional como particular, público y privado que permita una unificación de información; con miras a crear un sistema de información nacional de cáncer de mama.

3. Crear por parte del SSSA una red de Información Departamental con participación de los laboratorios de patología públicos y privados sobre cáncer de mama, a través de un suministro permanente y continuo de datos positivos de cáncer que permita mantener actualizadas las estadísticas y tomar conductas frente a dichos resultados.

4. Replicar esta investigación a nivel nacional como un estudio cooperativo que permita conocer la verdadera situación del cáncer de mama en el país y crear la red nacional de información.

5. Crear en cada Institución una Clínica de mama para un manejo multidisciplinario como centro de remisión y donde surjan campañas de educación e información que aporten a las estrategias nacionales para el diagnóstico precoz del cáncer mamario.

6. Incluir la exploración mamaria de rutina en el examen físico de toda mujer, que acuda a cualquier institución de salud.

7. Seleccionar las pacientes de riesgo para cáncer de mama e ingresarlas en un programa de seguimiento para el diagnóstico precoz y lograr así la disminución de la mortalidad.

8. Recomendamos como la Sociedad Americana de Cáncer y el Colegio Americano de Radiología, el uso de la mamografía como tamizaje en las pacientes de riesgo.

9. Diseñar programas educativos para la enseñanza del auto examen de mamas.

\section{Agradecimientos}

A cada uno de los Patólogos del Departamento de Antioquia por haber facilitado la revisión de sus archivos. Sin su aporte hubiera sido imposible esta investigación.

A la fundación Pedro Nel Cardona y al Servicio Seccional de Salud de Antioquia por su aporte económico y estímulo permanente a la investigación y permitir la aplicación de los resultados.

\section{BIBLIOGRAFIA}

1. Baker L. Breast cancer detection demostration project: five year summary report Ca-A. En: Cancer Journal for Clinicians. 1982; 32(4): 194-225.

2. Basset I., Mangikian V and Gold R. Mamography and breast cancer screening. En: Surgical Clinics of North America. 1990; 70(4): 775800 .

3. Celotio A., Calero F y Armas A. Fundamentos de Oncología ginecológica. [S.1.]: Ediciones Días de Santos, 1986; 625-737.

4. Cooper RA. Mamografía. En: Clínicas Obstétricas y Ginecológicas de Norte América. 1989; 4: 757-773.

5. Cortés CC. Observaciones sobre citología de seno. En: Revista Colombiana de Obstetricia y Ginecología. 1990; 51(2): 134-136.

6. Crichlow RW. Carcinoma of the male breast. En: Surgical Gynecology Obstetrics. 1972; 134: 1011-1015.

7. Crichlow RW and Galt S. Male breast cancer. En: Surgical Clinics of North America. 1990; 70(5): 1165-1177.

8. Donovan A. Bilateral breast cancer. En: The Surgical Clinics of North America. 1990; 70(5): 1141-1149.

9. Ellege R., Maguire W. Pronostic factors in breast cancer. En: Seminars in oncology. 1992; 19(3): 244-253.

10. Fisher E., Redmond C. Pathologic findings from the national surgical adjuvant breast and bowel proyects (NSABBP). En: Cancer 1990; 65: 2121-2128.

11. Fisher B. Frecuencia de las variedades histológicas de cáncer de mama. En: Clínicas Quirúrgicas de Norte América. 1978; 4: 705-735.

12. García VR et al. Estadio epidemiológico de cáncer de mama. En: Tokio Ginecology Practice. 1987; 46(8): 397.

13. González-Merlo J. Oncología Ginecológica. Bogotá: Salvat Editores, 1991; 379-542.

14. Haagensen CD. Enfermedades de la mama. Buenos Aires: Panamericana, 1987; 143-158.

15. Harris J et al. Spetial report the epidemiology and etiology of breast cancer. En: The New England Journal of Medicine. 1992; 327(6): 390-398.
16. Lynch H., Watson P., Linch J. Epidemiología y factores de riesgo de cáncer mamario. En:Clínicas Obstétricas y Ginecológicas de Norte América. 1989; 14: 741-749.

17. Martchant D. Frecuencia del cáncer de la mama. En: Clínicas Obstétricas y Ginecológicas de Norte América. 1982; 2: 412-420.

18. Moravia A. and Wynder E. Epidemiology and natural history of breast cancer: implications for body weight-breast cancer controversy. En: The Surgical Clinics of North America. 1990; 70(4): 739-752.

19. Morrison A. Review of evidence on the early detection and treatment of breast cancer. En: Cancer. 1989; 64(12): 2651-2660.

20. Pike M., Krailo $\mathrm{M}$ and Henderson B. Hormonal risk factors, breast tissue age and the age incidence of breast. En: Nature 1983; 303: 767 769.

21. Restrepo $\mathrm{H}$ et al. Epidemiología y control del cáncer de cuello uterino en América Latina y del Caribe. En: Boletín de la Oficina Sanitaria Panamericana. 1987; 102(6): 578-591.

22. Restrepo J. Punción aspiración de los tumores de mama y estudio citológico. En: Antioquia Médica. 1962; 13(2): 180-188.

23. Ropin V., Contesso G., Mouresse H. Medullary breast carcinoma. En: Cancer. 1988; 61: 2503-2510.

24. Siddigui T., Weiner R and Moreb J. Cancer of the male breast with prolonged survival. En: Cancer. 1988; 62: 1632-1638.

25. Sierra B., Díaz C. Masa de mama: comparación entre estudio histológico y citológico. CES: En: Medicina. 1988; 2(2): 105-107.

26. Silverberg E., Boaring C and Squires T. Cancer statites. 1990.

27. Update national breast and cancer early detection program July 1991July 1992. Morbility and Mortality. En: Weekly Report. 1992; 41(40): 739-743.

28. Wheller JE and Enterline HT. Lobular carcinoma of the breast in situ and infiltrating. En: Sommers SC (dir). Patholog Annual. New York: Appleton Century Crofts, 1976; 161 